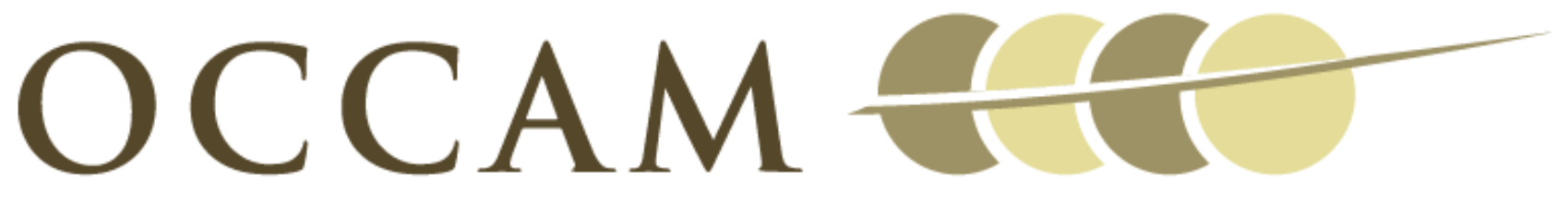

OXFORD CENTRE FOR COLLABORATIVE APPLIED MATHEMATICS

\author{
Report Number 09/33
}

Nematic liquid crystals : from Maier-Saupe to a continuum theory by

John M. Ball and Apala Majumdar

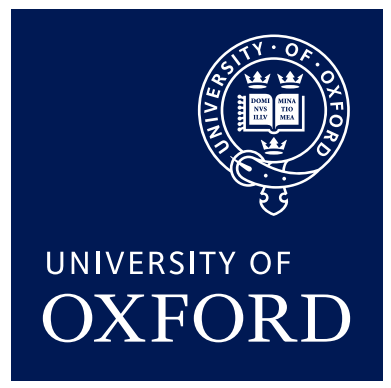

Oxford Centre for Collaborative Applied Mathematics Mathematical Institute 24 - 29 St Giles'

Oxford

OX1 3LB

England 



\title{
Nematic liquid crystals : from Maier-Saupe to a continuum theory
}

John M. Ball, Apala Majumdar*

July 1, 2009

\begin{abstract}
We define a continuum energy functional in terms of the meanfield Maier-Saupe free energy, that describes both spatially homogeneous and inhomogeneous systems. The Maier-Saupe theory defines the main macroscopic variable, the $\mathbf{Q}$-tensor order parameter, in terms of the second moment of a probability distribution function. This definition requires the eigenvalues of $\mathbf{Q}$ to be bounded both from below and above. We define a thermotropic bulk potential which blows up whenever the eigenvalues tend to these lower and upper bounds. This is in contrast to the Landau-de Gennes theory which has no such penalization. We study the asymptotics of this bulk potential in different regimes and discuss phase transitions predicted by this model.
\end{abstract}

\section{Introduction}

There are two commonly used approaches to the mathematical modelling of nematic liquid crystals. The first is the mean-field approach $[9,4]$. In

${ }^{*}$ Mathematical Institute, University of Oxford, 24-29 St.Giles, Oxford, OX1 3LB (ball@maths.ox.ac.uk, majumdar@maths.ox.ac.uk). 
the mean-field framework, the state of alignment of the nematic molecules is described by a probability distribution function $\rho$ on the unit sphere. The main macroscopic variable, the $\mathbf{Q}$-tensor order parameter, is defined in terms of the second moment of $\rho$ as shown below $[4,11]$

$$
\mathbf{Q}=\int_{S^{2}}\left(\mathbf{p} \otimes \mathbf{p}-\frac{1}{3} \mathbf{I}\right) \rho(\mathbf{p}) d \mathbf{p}
$$

and this definition immediately requires the $\mathbf{Q}$-tensor to be a symmetric, traceless $3 \times 3$ matrix whose eigenvalues $\left\{\lambda_{i}(\mathbf{Q})\right\}$ are constrained by the following inequalities $[7,10]$

$$
-\frac{1}{3} \leq \lambda_{i}(\mathbf{Q}) \leq \frac{2}{3} \quad i=1,2,3 ; \quad \sum_{i=1}^{3} \lambda_{i}(\mathbf{Q})=0 .
$$

In the rest of the paper we refer to (2) as physical constraints and to the range $\lambda_{i} \in\left(-\frac{1}{3}, \frac{2}{3}\right)$ as the physical regime.

The second approach is the phenomenological Landau-de Gennes theory $[4,11]$. The Landau-de Gennes theory also describes the state of a nematic liquid crystal by the macroscopic Q-tensor order parameter. Within the Landau-de Gennes theory, the Q-tensor order parameter is a symmetric, traceless $3 \times 3$ matrix with no a priori bounds on the eigenvalues and is often defined in terms of anisotropic macroscopic quantities such as the magnetic susceptibility. In the absence of surface energies and external fields, the Landau-de Gennes energy functional is given by

$$
I_{L G}[\mathbf{Q}]=\int f_{B}(\mathbf{Q})+w(\mathbf{Q}, \nabla \mathbf{Q}) d V
$$

Here $f_{B}$ is a thermotropic bulk potential that governs the preferred phase of the nematic liquid crystal - isotropic, uniaxial or biaxial - as function of the 
temperature and $w$ is an elastic energy density that penalizes spatial inhomogeneities. The function $f_{B}$ is a polynomial in the scalar invariants of the Q-tensor with material-dependent and temperature-dependent coefficients $[4,11,10]$

$$
f_{B}(\mathbf{Q})=\frac{1}{2} a(T) \operatorname{tr} \mathbf{Q}^{2}+\frac{1}{3} b \operatorname{tr} \mathbf{Q}^{3}+\frac{1}{4} c\left(\operatorname{tr} \mathbf{Q}^{2}\right)^{2}+\ldots
$$

and has no term that enforces the physical constraints on the eigenvalues in (2). The equilibrium (physically observable) configurations correspond either to global or local minimizers of the Landau-de Gennes energy subject to the imposed boundary conditions. We can make quantitative predictions about the order parameters (eigenvalues) of equilibrium configurations in both the spatially homogeneous and spatially inhomogeneous cases and these explicit estimates show that, since the Landau-de Gennes energy density has no forcing term to penalize configurations outside the physical regime, the equilibrium order parameters can take values outside the range (2) even for temeratures quite close to the nematic-isotropic transition temperature [10].

In this paper, we define a continuum energy functional in terms of the mean-field Maier-Saupe energy. Our approach is very similar to that in the paper by Katriel et.al [8] but we present our results in the continuum framework as opposed to the statistical mechanics framework. We account for both uniaxial and biaxial phases and include spatial inhomogeneities in the model. The key step is to define a new bulk potential $\psi_{B}$ that blows up whenever the eigenvalues approach the limiting values of either $-\frac{1}{3}$ or $\frac{2}{3}$ in (2) i.e. $\psi_{B}$ acts as an infinitely steep well that enforces the equilibrium order parameters to be physically realistic in both the spatially homogeneous and inhomogeneous cases. We show that $\psi_{B}$ exhibits a logarithmic divergence 
as the eigenvalues approach either $-\frac{1}{3}$ or $\frac{2}{3}$. We also study phase transitions within this framework and show that $\psi_{B}$ predicts a first-order nematicisotropic phase transition. Finally, we also consider an elastic energy density $w(\mathbf{Q}, \nabla \mathbf{Q})$ with four elastic constants. In this case the Landau-de Gennes energy $I_{L G}$ is in general unbounded from below, so that there is no global energy minimizer. However, this unphysical behaviour disappears if we use our bulk potential $\psi_{B}$ instead of the Landau-de Gennes bulk potential $f_{B}$.

\section{The Model}

For a spatially homogeneous system, the mean-field Maier-Saupe free energy is given by $[9,8,6]$ -

$$
I_{M S}[\rho]=T \int_{S^{2}} \rho(\mathbf{p}) \ln \rho(\mathbf{p}) d \mathbf{p}-\kappa|\mathbf{Q}|^{2}
$$

where $\rho$ is the probability distribution function for molecular orientations, $T$ is the absolute temperature, $\kappa$ is a constant related to the strength of the intermolecular interactions and $\mathbf{Q}$ is the normalized second moment of $\rho$ as in (1). We refer to the first integral term in (5) as the entropy term and to the second term as the intermolecular interaction term.

We define a bulk potential $\psi_{B}=\psi_{B}(\mathbf{Q})$ as follows:

$$
\psi_{B}(\mathbf{Q})=T \inf _{\rho \in \mathcal{A}_{\mathbf{Q}}} \int_{S^{2}} \rho(\mathbf{p}) \ln \rho(\mathbf{p}) d \mathbf{p}-\kappa|\mathbf{Q}|^{2}
$$

where

$$
\mathcal{A}_{\mathbf{Q}}=\left\{\rho: S^{2} \rightarrow \mathbb{R}, \rho \geq 0, \int_{S^{2}} \rho(\mathbf{p}) d \mathbf{p}=1 ; \mathbf{Q}=\int_{S^{2}}\left(\mathbf{p} \otimes \mathbf{p}-\frac{1}{3} \mathbf{I}\right) \rho(\mathbf{p}) d \mathbf{p}\right\}
$$

Thus, for a given $\mathbf{Q}$, we minimize the entropy term over all probability dis- 
tributions $\rho$ that have a fixed normalized second moment Q. Consequently, $\psi_{B}$ is a function of $\mathbf{Q}$ and not of $\rho$ (see Katriel et.al for a similar definition) and can describe both equilibrium and non-equilibrium liquid crystal configurations.

\section{The Bulk Potential}

Let

$$
f(\mathbf{Q})= \begin{cases}\inf _{\rho \in \mathcal{A}_{\mathbf{Q}}} \int_{S^{2}} \rho(\mathbf{p}) \ln \rho(\mathbf{p}) d \mathbf{p}, & \text { if } \lambda_{i}(\mathbf{Q}) \in\left(-\frac{1}{3}, \frac{2}{3}\right) \\ +\infty, & \text { otherwise }\end{cases}
$$

i.e. the minimization over $\mathcal{A}_{\mathbf{Q}}$ is only defined for those $\mathbf{Q}$-tensors which can be expressed as the normalized second moment of a probability distribution function $\rho$ and whose eigenvalues obey the physical constraints (2). We define $f(\mathbf{Q})$ to be unbounded (to be $+\infty$ ) for those $\mathbf{Q}$-tensors which are outside this physical regime. Then

$$
\psi_{B}(\mathbf{Q})=T f(\mathbf{Q})-\kappa|\mathbf{Q}|^{2}
$$

and the main aim of this analysis is to study the properties of the function $f(\mathbf{Q})$. We first present some preliminary facts about $f(\mathbf{Q})$ below.

Proposition 1. For a given $\mathbf{Q}$ with eigenvalues $\lambda_{i}(\mathbf{Q}) \in\left(-\frac{1}{3}, \frac{2}{3}\right)$, the minimization problem

$$
f(\mathbf{Q})=\inf _{\rho \in \mathcal{A}_{\mathbf{Q}}} \int_{S^{2}} \rho(\mathbf{p}) \ln \rho(\mathbf{p}) d \mathbf{p}
$$


has a unique minimizer $\rho^{*}$ in the class $\mathcal{A}_{\mathbf{Q}}$, where $\rho^{*}$ is given by

$$
\rho^{*}(\mathbf{p})=\frac{\exp \left(\sum_{i=1}^{3} \mu_{i} p_{i}^{2}\right)}{Z\left(\mu_{1}, \mu_{2}, \mu_{3}\right)}
$$

where $\mathbf{p}=\left(p_{1}, p_{2}, p_{3}\right) \in S^{2},\left\{\mu_{1}, \mu_{2}, \mu_{3}\right\}$ are Lagrange multipliers (unique up to the addition of an arbitrary constant) associated with the constraint $\mathbf{Q}=\int_{S^{2}}\left(\mathbf{p} \otimes \mathbf{p}-\frac{1}{3} \mathbf{I}\right) \rho(\mathbf{p}) d \mathbf{p}\left(\right.$ see the definition of $\mathcal{A}_{\mathbf{Q}}$ in $\left.(7)\right), Z\left(\mu_{1}, \mu_{2}, \mu_{3}\right)$ is the partition function defined by

$$
Z\left(\mu_{1}, \mu_{2}, \mu_{3}\right)=\int_{S^{2}} \exp \left(\sum_{i=1}^{3} \mu_{i} p_{i}^{2}\right) d \mathbf{p}
$$

and

$$
\begin{aligned}
& \frac{1}{Z} \frac{\partial Z}{\partial \mu_{1}}=\lambda_{1}+\frac{1}{3} \\
& \frac{1}{Z} \frac{\partial Z}{\partial \mu_{2}}=\lambda_{2}+\frac{1}{3} \\
& \frac{1}{Z} \frac{\partial Z}{\partial \mu_{3}}=\lambda_{3}+\frac{1}{3}
\end{aligned}
$$

Proof. The proof of this proposition is quite technical. The key steps are to show that (i) $\mathcal{A}_{\mathbf{Q}}$ is nonempty for any $\mathbf{Q}$ whose eigenvalues obey the physical constraints (2), (ii) $f(\mathbf{Q})$ is a strictly convex function of the $\mathbf{Q}$-tensor and (iii) the Euler-Lagrange equations hold for the minimization problem (10). We point out that the relations (13) implicitly define the Lagrange multipliers $\mu_{i}$ as functions of $\mathbf{Q}$ up to the addition of an arbitrary constant to each $\mu_{i}$, i.e. the differences $\mu_{i}-\mu_{j}$ are unique. The proof is similar in spirit to the methods in $[8,6]$ and we omit the details here for brevity.

Proposition 2. The function $f(\mathbf{Q}) \rightarrow+\infty$ whenever one of the eigenvalues approaches the lower limiting value of $-\frac{1}{3}$ in $(2)$ i.e. when $\lambda_{i}(\mathbf{Q}) \rightarrow-\frac{1}{3}^{+}$ 
for some $i=1,2,3$.

Proof. For definiteness, let us assume that $\lambda_{1}(\mathbf{Q}) \rightarrow-\frac{1}{3}^{+}$. We recall that this lower bound describes a liquid crystal configuration where the nematic molecules are everywhere perpendicular to the corresponding eigenvector $[7,10]$. In this limit, the probability distribution function $\rho$ is necessarily singular in the sense that it is supported on the unit circle perpendicular to this eigenvector. Therefore, $\ln \rho$ diverges and consequently, $f(\mathbf{Q}) \rightarrow \infty$ or $f(\mathbf{Q})$ becomes unbounded. Analogous remarks apply if $\lambda_{i}(\mathbf{Q}) \rightarrow \frac{2}{3}$ for some $i=1,2,3$ as this describes the physically unrealistic state of perfect alignment along the corresponding eigenvector. For technical details, see $[2]$.

Given the minimizing $\rho^{*}$ in (11), we have from (9) and (12) that

$$
\begin{aligned}
& \psi_{B}(\mathbf{Q})=T \sum_{i=1}^{3} \mu_{i}\left(\lambda_{i}(\mathbf{Q})+\frac{1}{3}\right)-T \ln Z\left(\mu_{1}, \mu_{2}, \mu_{3}\right)-\kappa|\mathbf{Q}|^{2} \\
& =T\left(\mu_{1}-\mu_{3}\right)\left(\lambda_{1}+\frac{1}{3}\right)+T\left(\mu_{2}-\mu_{3}\right)\left(\lambda_{2}+\frac{1}{3}\right)-\kappa|\mathbf{Q}|^{2}- \\
& -T \ln \left[\int_{S^{2}} \exp \left(\left(\mu_{1}-\mu_{3}\right) p_{1}^{2}+\left(\mu_{2}-\mu_{3}\right) p_{2}^{2}\right) d \mathbf{p}\right]
\end{aligned}
$$

In (15), we have used the explicit form of the partition function $Z$ in (12). From the definition of $\psi_{B}$ in (9), it is evident that $\psi_{B}(\mathbf{Q}) \rightarrow+\infty$ if and only if $f(\mathbf{Q}) \rightarrow \infty$ and from the semi-explicit expression in (15), $\psi_{B} \rightarrow+\infty$ if and only if either $\mu_{1}-\mu_{3} \rightarrow \pm \infty$ or $\mu_{2}-\mu_{3} \rightarrow \pm \infty$ or both. Since the function $f(\mathbf{Q})$ diverges whenever $\lambda_{i}(\mathbf{Q}) \rightarrow-\frac{1}{3}^{+}$for some $i=1 \ldots 3$, we deduce that

Corollary 1. The limits $\mu_{1}-\mu_{3} \rightarrow \pm \infty$ or $\mu_{2}-\mu_{3} \rightarrow \pm \infty$ correspond to one or more of the eigenvalues approaching the lower bound in (2) i.e. they describe physically unrealistic configurations. 
As an illustrative example, let us consider the arbitrary uniaxial state $\left(\lambda_{1}, \lambda_{2}, \lambda_{3}\right)=(\lambda, \lambda,-2 \lambda)$ where $\lambda$ is the non-zero degenerate eigenvalue. From (11), the minimizing $\rho^{*}$ in given by

$$
\rho^{*}(\theta, \phi)=\frac{\exp \left(\mu_{1} \sin ^{2} \theta+\mu_{3} \cos ^{2} \theta\right)}{Z\left(\mu_{1}, \mu_{3}\right)}
$$

where $(\theta, \phi)$ are spherical polar coordinates on the unit sphere, $Z\left(\mu_{1}, \mu_{3}\right)=$ $2 \pi \exp \left(\mu_{3}\right) \int_{-1}^{1} \exp \left(\left(\mu_{1}-\mu_{3}\right)\left(1-\nu^{2}\right)\right) d \nu$ is the partition function and

$$
\frac{1}{Z} \frac{\partial Z}{\partial \mu_{3}}=\frac{1}{2\left(\mu_{1}-\mu_{3}\right)}-\frac{2 \pi \exp \left(\mu_{3}\right)}{\mu_{1}-\mu_{3}} \frac{1}{Z\left(\mu_{1}, \mu_{3}\right)}=-2 \lambda+\frac{1}{3}
$$

(We note that (16)-(17) is the same as the uniaxial formulation in Katriel et.al [8] and that $Z\left(\mu_{1}, \mu_{3}\right)$ can be explicitly expressed in terms of the Dawson's integral although we will not need the explicit expression for this discussion.)

The question of interest is - what do the limits $\mu_{1}-\mu_{3} \rightarrow \pm \infty$ correspond to in terms of the eigenvalue $\lambda$ ? It is straightforward to verify from (17) that if $\mu_{1}-\mu_{3} \rightarrow+\infty$, then

$$
\lambda=\frac{1}{6}-\frac{1}{4\left(\mu_{1}-\mu_{3}\right)}+\mathrm{o}\left(\frac{1}{\mu_{1}-\mu_{3}}\right)
$$

(o $\left(\frac{1}{\mu_{1}-\mu_{3}}\right)$ includes terms which are much smaller than $\frac{1}{\mu_{1}-\mu_{3}}$ in this limit) and

$$
\psi_{B}(\mathbf{Q})=T C_{1} \ln \left(\mu_{1}-\mu_{3}\right)-\frac{\kappa}{6}+T C_{2}+\frac{\kappa}{2\left(\mu_{1}-\mu_{3}\right)}+o\left(\frac{1}{\mu_{1}-\mu_{3}}\right)
$$

where $C_{1}$ and $C_{2}$ are positive constants independent of $\mu_{1}, \mu_{3}$ and $\kappa$. Thus, the limit $\mu_{1}-\mu_{3} \rightarrow+\infty$ corresponds to the oblate uniaxial state $\left(\lambda_{1}, \lambda_{2}, \lambda_{3}\right)=$ $\left(\frac{1}{6}, \frac{1}{6},-\frac{1}{3}\right)$ and the bulk potential $\psi_{B}$ exhibits a logarithmic divergence in 
this limit.

Similarly, if $\mu_{1}-\mu_{3} \rightarrow-\infty$, then we can carry out standard asymptotic estimates using Laplace's method [3] to show that

$$
\lambda=-\frac{1}{3}+\frac{1}{4\left|\mu_{1}-\mu_{3}\right|}+o\left(\frac{1}{\left|\mu_{1}-\mu_{3}\right|}\right)
$$

in this limit and

$$
\psi_{B}(\mathbf{Q})=T B_{1} \ln \left|\mu_{1}-\mu_{3}\right|-\frac{2 \kappa}{3}+T B_{2}+\frac{\kappa}{\left|\mu_{1}-\mu_{3}\right|}+o\left(\frac{1}{\left|\mu_{1}-\mu_{3}\right|}\right)
$$

as $\mu_{1}-\mu_{3} \rightarrow-\infty$, where $B_{1}, B_{2}$ are positive constants independent of $\mu_{1}, \mu_{3}$ and $\kappa$. Therefore, the limit $\mu_{1}-\mu_{3} \rightarrow-\infty$ corresponds to the prolate uniaxial state $\left(\lambda_{1}, \lambda_{2}, \lambda_{3}\right)=\left(-\frac{1}{3},-\frac{1}{3}, \frac{2}{3}\right)$ and $\psi_{B}$ exhibits a logarithmic divergence in this case too.

These calculations can be generalized to the biaxial case where $Z\left(\mu_{1}, \mu_{2}, \mu_{3}\right)$ is given by (12) and we are interested in the limits $\mu_{1}-\mu_{3} \rightarrow \pm \infty$ and $\mu_{2}-\mu_{3} \rightarrow \pm \infty$. The main results are:

1. $\mu_{1}-\mu_{3}, \mu_{2}-\mu_{3} \rightarrow+\infty$ corresponds to a $\mathbf{Q}$-tensor with

$$
\lambda_{3}+\frac{1}{3}=C\left\{\frac{1}{\mu_{1}-\mu_{3}}+\frac{1}{\mu_{2}-\mu_{3}}\right\}+\mathrm{o}\left(\frac{1}{\mu_{1}-\mu_{3}}+\frac{1}{\mu_{2}-\mu_{3}}\right)
$$

where $C$ is a positive constant.

2. $\mu_{1}-\mu_{3} \rightarrow+\infty, \mu_{2}-\mu_{3} \rightarrow-\infty$ corresponds to a prolate uniaxial state $\left(\lambda_{1}, \lambda_{2}, \lambda_{3}\right)=\left(\frac{2}{3},-\frac{1}{3},-\frac{1}{3}\right)$

3. $\mu_{1}-\mu_{3} \rightarrow-\infty, \mu_{2}-\mu_{3} \rightarrow+\infty$ corresponds to the prolate uniaxial state $\left(\lambda_{1}, \lambda_{2}, \lambda_{3}\right)=\left(-\frac{1}{3}, \frac{2}{3},-\frac{1}{3}\right)$

4. $\mu_{1}-\mu_{3}, \mu_{2}-\mu_{3} \rightarrow-\infty$ corresponds to the prolate uniaxial state 


$$
\left(\lambda_{1}, \lambda_{2}, \lambda_{3}\right)=\left(-\frac{1}{3},-\frac{1}{3}, \frac{2}{3}\right)
$$

Cases with $\mu_{1}-\mu_{3} \rightarrow \pm \infty$ and $\mu_{2}-\mu_{3}=O(1)$ or $\mu_{1}-\mu_{3}=O(1)$ and $\mu_{2}-\mu_{3} \rightarrow \pm \infty$ can be treated in an analogous manner and, in all cases, the bulk potential $\psi_{B}$ exhibits a logarithmic divergence. We can summarize these computations as follows.

Corollary 2. Let $\mu_{i}-\mu_{j} \rightarrow \pm \infty$ in the definition of $Z\left(\mu_{1}, \mu_{2}, \mu_{3}\right)$ in (12), for a pair of Lagrange multipliers $\mu_{i}$ and $\mu_{j}$. Then one or more of the eigenvalues approach the lower bound of $-\frac{1}{3}$ in (2) and the leading order asymptotics of $\psi_{B}$ is given by

$$
\psi_{B} \sim T C \ln \left|\mu_{i}-\mu_{j}\right|
$$

where $C$ is a positive constant.

\section{Nematic-Isotropic Phase Transition}

In this section, we show that the bulk potential $\psi_{B}$ predicts a first-order nematic-isotropic phase transition as in the Landau-de Gennes theory. Our first result concerns the nature of stationary points of $\psi_{B}$.

Proposition 3. Every stationary point of the bulk potential

$$
\begin{aligned}
& \psi_{B}(\mathbf{Q})=T f(\mathbf{Q})-\kappa|\mathbf{Q}|^{2} \\
& \quad=T \sum_{i=1}^{3} \mu_{i}(\mathbf{Q})\left(\lambda_{i}(\mathbf{Q})+\frac{1}{3}\right)-T \ln Z\left(\mu_{1}, \mu_{2}, \mu_{3}\right)-\kappa|\mathbf{Q}|^{2}
\end{aligned}
$$

must have at least two equal eigenvalues.

This implies that every stationary point of $\psi_{B}$ must be either uniaxial or isotropic. The proof is very similar to the methods in [6] and we omit the 
details here for brevity.

Let $\left(\lambda_{1}, \lambda_{2}, \lambda_{3}\right)=(\lambda, \lambda,-2 \lambda)$ be a uniaxial stationary point of $\psi_{B}$ in (9). The corresponding $\mathbf{Q}$-tensor is

$$
\mathbf{Q}_{u}=S\left(\mathbf{e}_{3} \otimes \mathbf{e}_{3}-\frac{1}{3} \mathbf{I}\right)
$$

where $S=-3 \lambda$ and $\mathbf{e}_{3}$ is the distinguished eigenvector (with eigenvalue $-2 \lambda)$. Then the stationarity condition $d \psi_{B} / d \lambda=0$ coupled with the relations (17) necessarily implies that

$$
\mu_{1}-\mu_{3}=6 \kappa \lambda
$$

Without going into technical details, (26) enables us to obtain an explicit expression for $\psi_{B}\left(\mathbf{Q}_{u}\right)$, where $\mathbf{Q}_{u}$ is a stationary point by assumption, and explicitly compute the stationary values of $\lambda$ (or equivalently the stationary values of the uniaxial order parameter $S$ ) within this framework. There are precisely three stationary values of $\lambda$ :

$$
\begin{aligned}
& \lambda=0 \\
& \lambda_{ \pm}(T)=\frac{-1 \pm \sqrt{9-\frac{30 T}{\kappa}}}{12}
\end{aligned}
$$

where $\lambda_{ \pm}(T)$ correspond to the ordered nematic phases and $\lambda_{-}(T)$ is the stable nematic stationary point. As $T \rightarrow 0, \lambda_{-}(T) \rightarrow-\frac{1}{3}$ and the corresponding value of $S=-3 \lambda_{-}(T) \rightarrow 1$ in this limit. Similarly, $\lim _{T \rightarrow 0} \lambda_{+}(T) \rightarrow \frac{1}{6}$ and therefore $S=-3 \lambda_{+}(T) \rightarrow-\frac{1}{2}$ in this limit.

As in the Landau-de Gennes theory, there are precisely three character- 
istic values of $T, T_{1}<T_{2}<T_{3}$ within this model -

$$
\begin{aligned}
& T_{1}=\frac{3 \kappa}{10}, \text { above which nematic states are not defined } \\
& T_{2}: \psi_{B}(0)=\psi_{B}\left(\lambda_{-}\left(T_{2}\right)\right) \\
& T_{3}=\frac{4 \kappa}{15} \text {, below which the isotropic state } \lambda=0 \text { loses its stability. }
\end{aligned}
$$

We note that $T_{2}$ is the analogue of the nematic-isotropic transition temperature and that the equilibrium uniaxial order parameter at the transition point $S_{N I}=-3 \lambda_{-}\left(T_{2}\right)$ lies in the range $\frac{1}{4}<S_{N I}<\frac{1}{2}$. Thus, this model predicts a first-order nematic-isotropic phase transition whilst respecting the physical constraints (2).

\section{Spatial Inhomogeneities}

Having studied the bulk potential $\psi_{B}$ in some detail, we next include spatial inhomogeneities into the model. We work within the one-constant approximation for the elastic energy density. Then the corresponding energy functional is

$$
E[\mathbf{Q}]=\int_{\Omega} \psi_{B}(\mathbf{Q})+L|\nabla \mathbf{Q}|^{2} d V
$$

where $\mathbf{Q}=\mathbf{Q}(\mathbf{x}), \Omega$ is a smooth, simply-connected domain in three-dimensional space and $L$ is an elastic constant. The equilibrium (physically observable) configurations are given either by global or local minimizers of this energy subject to the imposed boundary conditions.

Theorem 1. Let $\mathbf{Q}^{*}$ be a global minimizer of $E[\mathbf{Q}]$ subject to the fixed boundary condition (strong anchoring), $\mathbf{Q}=\mathbf{Q}_{b}$ on $\partial \Omega$. We take the boundary condition $\mathbf{Q}_{b}$ to be physically realistic in the sense $-\frac{1}{3}+\epsilon_{0}<\lambda_{i}\left(\mathbf{Q}_{b}\right)<\frac{2}{3}-\epsilon_{0}$ for $i=1,2,3$, for some $\epsilon_{0}>0$, or equivalently $\psi_{B}\left(\mathbf{Q}_{b}\right)$ is bounded. Then 
the eigenvalues, $\lambda_{i}\left(\mathbf{Q}^{*}\right)$, of $\mathbf{Q}^{*}$ are constrained by the inequalities

$$
-\frac{1}{3}+\epsilon<\lambda_{i}\left(\mathbf{Q}^{*}\right)<\frac{2}{3}-\epsilon \quad i=1,2,3
$$

for some $\epsilon>0$ i.e. the eigenvalues of $\mathbf{Q}^{*}$ obey the physical constraints in $(2)$.

Remark: Since $\psi_{B}$ blows up whenever the physical constraints (2) are violated, it is evident that a global energy minimizer $\mathbf{Q}^{*}$ must obey $-\frac{1}{3}<$ $\lambda_{i}\left(\mathbf{Q}^{*}\right)<\frac{2}{3}$ almost everywhere on $\Omega$, except possibly for a set of zero volume. The force of the above Theorem lies in the fact that $\lambda_{i}\left(\mathbf{Q}^{*}\right)$ remain bounded away from the limiting values, $-\frac{1}{3}$ or $\frac{2}{3}$, everywhere in $\Omega$ i.e. they cannot approach the limiting values arbitrarily closely. While this is plausible, there are well-known examples in the calculus of variations in which minimizers have defects where the energy density (the analogue of $\psi_{B}$ in our case) blows up.

Proof. The proof of this theorem follows from a maximum principle approach [5] and the details are omitted here for brevity.

\section{Example with Four Elastic Constants}

We have considered the one-constant approximation for the elastic energy density $w(\mathbf{Q}, \nabla \mathbf{Q})$ in Section 5. There are more general forms of the elastic energy density such as [11]

$$
w(\mathbf{Q}, \nabla \mathbf{Q})=L_{1}|\nabla \mathbf{Q}|^{2}+L_{2} Q_{i k, j} Q_{i j, k}+L_{3} Q_{i j, j} Q_{i k, k}+L_{4} Q_{l k} Q_{i j, k} Q_{i j, l}
$$

where $\mathbf{Q}=\left(Q_{i j}\right), Q_{i j, k}=\frac{\partial Q_{i j}}{\partial x_{k}}$ and $L_{1}, \ldots, L_{4}$ are material-dependent elastic constants. We present an example with four elastic constants in the Landau- 
de Gennes framework and show that if $L_{4} \neq 0$, then the energy is unbounded from below.

Proposition 4. For any boundary conditions, if $L_{4} \neq 0$, then the Landau-de Gennes energy

$$
I_{L G}[\mathbf{Q}]=\int_{\Omega} f_{B}(\mathbf{Q})+w(\mathbf{Q}, \nabla \mathbf{Q}) d V
$$

where $f_{B}(\mathbf{Q})$ is as in (4) and $w(\mathbf{Q}, \nabla \mathbf{Q})$ is as in $(31)$, is unbounded from below (that is, there exists a $\mathbf{Q}$ such that $I_{L G}[\mathbf{Q}] \rightarrow-\infty$ ).

Proof. Choose any $\mathbf{Q}$ satisfying the boundary conditions and redefine it by multiplying it by a smooth function $\phi$, which equals one in a neighbourhood of the domain boundary and is zero in some ball $B \subset \Omega$, which we can take to be the unit ball $B(0,1)$.Thus $\mathbf{Q}$ vanishes on $B$ and we are free to alter $\mathbf{Q}$ in $B$ subject to $\left.\mathbf{Q}\right|_{\partial B}=0$. We alter $\mathbf{Q}$ in $B$ so that $I_{L G}[\mathbf{Q}]$ is arbitrarily large and negative. We set

$$
\mathbf{Q}(\mathbf{x})=\eta(|\mathbf{x}|)\left[\frac{\mathbf{x}}{|\mathbf{x}|} \otimes \frac{\mathbf{x}}{|\mathbf{x}|}-\frac{1}{3} \mathbf{I}\right], \eta(1)=0
$$

Then

$$
|\nabla \mathbf{Q}|^{2}=Q_{i j, k} Q_{i j, k}=\frac{2}{3} \eta^{\prime 2}+\frac{4}{|\mathbf{x}|^{2}} \eta^{2}
$$

and

$$
Q_{l k} Q_{i j, k} Q_{i j, l}=\frac{4}{9} \eta\left(\eta^{\prime 2}-\frac{3}{|\mathbf{x}|^{2}} \eta^{2}\right)
$$

Therefore, since $\left|Q_{i k, j} Q_{i j, k}\right|$ and $\left|Q_{i j, j} Q_{i k, k}\right|$ are bounded by const. $\times|\nabla \mathbf{Q}|^{2}$,

$$
\begin{aligned}
& I_{L G}[\mathbf{Q}] \leq 4 \pi C \int_{0}^{1} r^{2}\left[f_{B}(\mathbf{Q})+\frac{2}{3} \eta^{\prime 2}+\frac{4}{|\mathbf{x}|^{2}} \eta^{2}\right] d r \\
& +4 \pi \int_{0}^{1} \frac{4 L_{4}}{9} \eta\left(\eta^{\prime 2}-\frac{3}{|\mathbf{x}|^{2}} \eta^{2}\right) r^{2} d r+\int_{\Omega \backslash B} f_{B}(\mathbf{Q})+w(\mathbf{Q}, \nabla \mathbf{Q}) d V
\end{aligned}
$$


where $r=|\mathbf{x}|$ and $C$ is a positive constant. We define

$$
\eta(r)= \begin{cases}\eta_{0}(2+\sin k r), & 0<r<\frac{1}{2}, \\ 2 \eta_{0}\left(2+\sin \frac{k}{2}\right)(1-r), & \frac{1}{2}<r<1 .\end{cases}
$$

Explicit computations now show that by choosing $\eta_{0}$ to be sufficiently negative $\left(\eta_{0}<0,\left|\eta_{0}\right|>>1\right)$ and $k$ to be sufficiently large and positive, $I_{L G}[\mathbf{Q}]$ can be made arbitrarily large and negative.

The above proposition implies in particular that there is no global minimizer for the Landau-de Gennes energy in the presence of the given cubic term in the elastic energy density. On the other hand, for our modified energy functional

$$
E[\mathbf{Q}]=\int_{\Omega} \psi_{B}(\mathbf{Q})+w(\mathbf{Q}, \nabla \mathbf{Q}) d V
$$

it is possible to prove the existence of global minimizers when $L_{4} \neq 0$, under suitable hypotheses on the elastic constants $L_{i}$. This is because the eigenvalue constraints (2) allow one to estimate the cubic term in terms of the quadratic ones (see [2]) and consequently, $E[\mathbf{Q}]$ remains bounded from below.

\section{Acknowledgements}

A. Majumdar is supported by Award No. KUK-C1-013-04, made by King Abdullah University of Science and Technology (KAUST) to the Oxford Centre for Collaborative Applied Mathematics. J.M. Ball is supported by EPSRC grants EP/E010288/1 and EP/E035027/1. The authors gratefully acknowledge helpful discussions with Geoffrey Luckhurst, Peter Palffy- 
Muhoray, Valery Slastikov and Tim Sluckin.

\section{References}

[1] J. M. Ball, Graduate lecture course "Mathematical theories of liquid crystals", 2007.

[2] J. M. Ball and A. Majumdar, Energy minimization and constraints on the eigenvalues of the $\mathbf{Q}$-tensor for nematic liquid crystals, in preparation 2009 .

[3] C. Bender and S. Orzag, Advanced Mathematical Methods for Scientists and Engineers: Asymptotic Methods and Perturbation Theory. McGraw Hill, 1978.

[4] P. G. De Gennes, The physics of liquid crystals. Oxford, Clarendon Press, 1974 .

[5] L. Evans, Partial Differential Equations. American Mathematical Society, Providence, 1998.

[6] I. Fatkullin and V. Slastikov, Critical points of the Onsager functional on a sphere. Nonlinearity, 18, 2565-2580 (2005).

[7] M. G. Forest, Q. Wang and H. Zhou, Homogeneous pattern selection and director instabilities of nematic liquid crystal polymers induced by elongational flows. Physics of Fluids, 12, no. 3, 490-498 (2000).

[8] J. Katriel, G.F. Kventsel, G.R. Luckhurst and T.J. Sluckin, Free Energies in the Landau and Molecular Field Approaches. Liquid Crystals 1, 337 -355 (1986). 
[9] W. Maier and A. Saupe, A simple molecular statistical theory of the nematic crystalline-liquid phase. I Z Naturf. a 14, 882-889 (1959).

[10] A. Majumdar, Equilibrium order parameters of liquid crystals in the Landau-de Gennes theory. Pre-print: http://arxiv.org/abs/0808.1870.

[11] N. J. Mottram and C. Newton, Introduction to Q-tensor Theory. University of Strathclyde, Department of Mathematics, Research Report, 10 (2004).

[12] P. Palffy-Muhoray, Lecture notes on Liquid Crystal Materials, 2004. Private communication. 


\section{RECENT REPORTS}

07/09 Solving the Coupled System Improves Computational Efficiency of the Bidomain Equations

Southern

Plank

Vigmond

Whiteley

08/09 Model reduction using a posteriori analysis

Whiteley

09/09 Equilibrium Order Parameters of Liquid Crystals in the LaudauDe Gennes Theory

Majumdar

10/09 Landau-De Gennes theory of nematic liquid crystals: the OseenFrank limit and beyond

Majumdar

Zarnescu

11/09 A Comparison of Numerical Methods used for Finite Element Modelling of Soft Tissue Deformation

Pathmanathan

Gavaghan

Whiteley

12/09 From Individual to Collective Behaviour of Unicellular Organisms: Recent Results and Open Problems

Xue

Othmer

Erban

13/09 Stochastic modelling of reaction-diffusion processes: algorithms

Erban for bimolecular reactions

Chapman

14/09 Chaste: a test-driven approach to software development for physiological modelling

15/09 Block triangular preconditioners for PDE constrained optimization

Pitt-Francis et al.

Rees

Stoll

16/09 From microscopic to macroscopic descriptions of cell migration on growing domains

Baker

Yates

Erban

17/09 The Influence of Gene Expression Time Delays on GiererMeinhardt Pattern Formation Systems

Seirin Lee

Gaffney

Monk

18/09 Analysis of a stochastic chemical system close to a sniper bifurca-

Erban et al. tion of its mean field model

19/09 On the existence and the applications of modified equations for stochastic differential equations

20/09 Pebble bed: reflector treatment and pressure velocity coupling

21/09 A finite difference method for free boundary problems

Charpin et al. Fornberg 
22/09 Tangent unit-vector fields: nonabelian homotopy invariants and the Dirichlet energy

Majumdar

Robbins

Zyskin

23/09 Morphological instability of a nonequilibrium icecolloid interface

Peppin

Majumdar

Wettlaufer

24/09 The effect of polar lipids on tear film dynamics

Aydemir

Breward

Witelski

25/09 Preconditioning for active set and projected gradient methods as

Stoll semi-smooth Newton methods for PDE-constrained optimization with control constraints

26/09 Functional differential equations arising in cell-growth

Wake

Begg

27/09 A Cell Growth Model Revisited

Derfel van Brunt Wake

28/09 Quasi-steady state reduction of molecular motor-based models of directed intermittent search

Newby

Bressloff

29/09 All-at-once preconditioning in PDE-constrained optimization

Rees

Stoll

Wathen

30/09 An hp-Local Discontinuous Galerkin method for Parabolic Pani Integro-Differential Equations

Yadav

31/09 Stochastic neural field theory and the system-size expansion

Bressloff

32/09 A Hamiltonian Krylov-Schur-type method based on the symplectic Lanczos process

Benner

Faßbender

Stoll

Copies of these, and any other OCCAM reports can be obtained from:

Oxford Centre for Collaborative Applied Mathematics Mathematical Institute

24 - 29 St Giles'

Oxford

OX1 3LB

England

www.maths.ox.ac.uk/occam 